\title{
Ludocore: A Logical Game Engine for Modeling Videogames
}

\author{
Adam M. Smith, Mark J. Nelson, and Michael Mateas
}

\begin{abstract}
LUDOCORE is a logical "game engine", linking game rules as reasoned about by game designers to the formal logic used by automated reasoning tools in AI. A key challenge in designing this bridge is engineering a concise, safe, and flexible representation that is compatible with the semantics of the games that logical models created with our engine intend to represent.

Building on the event calculus, a formalism for reasoning about state and events over time, and a set of common structures and idioms used in modeling games, we present a tool that is capable of generating gameplay traces that illustrate the game's dynamic behavior. It supports incremental modeling of player and non-player entities in the game world, modification of game rules without extensive non-local changes, and exploratory temporal and structural queries. In addition, its logical models can support play as real-time, graphical games with minimal user-interface description.
\end{abstract}

\section{INTRODUCTION}

While the term videogame brings to mind graphics, sounds, and story worlds, at the core of every game is a formal rule system. We are interested in declaratively modeling these games so that the emergent properties of their rule systems can be understood. The tools of logicist AI hold promise for bringing such properties to light, but have not been used for design or analysis of videogames, in part because of a mismatch between how designers think of their rule systems and the way logical specifications are usually written. We propose the use of a logical "game engine" to ease and accelerate the modeling of game worlds in formal logic. Our engine, LUDOCORE, provides a set of primitives and abstractions that link game-level concepts to the first-order logic understood by AI reasoning tools. Specifically, our engine supports automatically generating gameplay traces, integrated player modeling, and both temporal and structural queries. Finally, games modeled in LUDOCORE can be directly read in, without compilation or translation, to a custom, prototyping-focused game engine (described in more detail elsewhere [1]), allowing them to be played as real-time, graphical games (an example, using visual elements styled after those used in paper prototyping, is shown in Figure 1).

An existing way to represent purely abstract games in logic is GDL, the game description language [2]. GDL was designed to specify games for the general gameplaying competition [3], in which computers competitively play unfamiliar, turn-based games. Thus, the language itself was designed primarily to write infrequently-edited specifications to be read and understood by computer players as statetransition systems. In contrast, when modeling videogames,

The authors are with the Expressive Intelligence Studio at the University of California, Santa Cruz (email: amsmith@soe.ucsc.edu, mnelson@cc.gatech.edu, michaelm@soe.ucsc.edu).

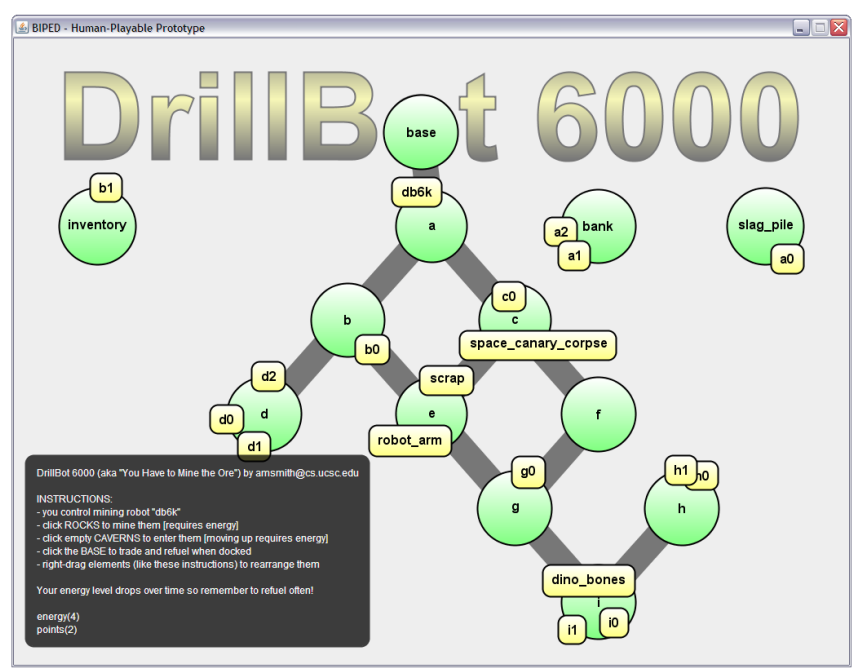

Fig. 1. Example of a playable version of a game specified in LUDOCORE, within a game engine based on paper-prototyping-style graphical elements. An automatically generated gameplay trace for this game is shown in Figure 3.

designers are much more concerned with the ease with which they can incrementally build up a game's rule system through a series of experimental tweaks [4, p. 14]. LUDOCORE supports this kind of modeling, at least for technically savvy designer-programmers.

Our iterative-design motivation also contrasts with the goals of formal verification. While verification attempts to prove that a set of desired properties hold, it is not clear during a game's prototyping phase which properties are even desirable. In fact, coming to understand what properties a game has, and which ones would be desirable, is the main challenge.

Our bridge from games to formal logic is based on the event calculus, a logical formalism commonly associated with commonsense reasoning. In previous research [5], we proposed that the event calculus is an attractive basis on which to build informative, queryable prototypes. Since then, in building games with the event calculus, we encountered various design patterns and idioms, common to all games; it is this experience which prompted the design of LUDOCORE.

\section{Building Games on the Event Calculus}

Standard game-design texts discuss a game's mechanics in terms of objects with dynamic properties (the state of the game), and triggered behaviors (events and conditional state updates) [6, p. 295] [4, p. 112]. While state and events can be modeled with several formalisms, or even ad-hoc logical encodings, a common challenge is the well known frame 
problem, commonly solved by adding frame axioms. In a language like GDL, frame axioms explicitly declare when state doesn't change from turn to turn. An alternate proposed solution is based on the commonsense law of inertia [7], which reads that states retain their values until an event changes them. This mirrors the usual game-programming assumption that variables stay set to the same value until changed by an active behavior.

Given our desire to model state and events, and avoid frame axioms that are tedious to maintain, the event calculus (EC) is a natural choice. Additionally, there is a large body of literature discussing the use of EC as a practical knowledge representation, e.g. Mueller's book [8] on applying it to commonsense reasoning problems involving interaction of objects over time, for which inertial state is the common case.

The discrete event calculus [9] is based on fluents (predicates whose truth values vary over time) and events, which happen at particular integer-valued timepoints and can change the truth values of fluents. Its key predicates are: happens $(E, T)$, which says that an event happens at a timepoint; holds_at $(F, T)$, which says that a fluent is true at a timepoint; and initiates $(E, F, T)$ and terminates $(E, F, T)$, which map event occurrences to changes in fluent truth values. In addition to state that does not change without cause (inertial fluents), the circumscription used in the event calculus implies that events have no effects besides those that can be derived. Together, these two kinds of default reasoning give the event calculus elaboration tolerance [10], the ability to modify a knowledge representation without re-engineering it, because new assertions override defaults.

To realize EC in a computational setting, we use answerset programming (ASP), an approach proposed in unpublished notes accompanying Mueller's book [8], ${ }^{1}$ which Kim et al. [11] proved preserves the expected EC semantics. Answer sets are sets of literals that represent acceptable beliefs in an abstract world [12]. When applied to programs in the discrete event calculus, these answer sets amount to assertions about what was true and what happened at each timepoint. Further, for games modeled in EC, the narrative of events amounts to what we call a gameplay trace. While there are more direct ways to extract a single trace from a game, ASP does not forward-simulate a game, but rather reasons abstractly about the space of possible executions, which is far more flexible.

The combination of EC+ASP is an interesting tool for game-related AI because ASP provides fast inference to models of a game's execution, and EC is a solid knowledge representation for abstract worlds. Together they facilitate generating traces from concise declarative descriptions. This trace inference is in fact more expressive than forward-search based methods such as Monte Carlo rollouts; for example, it can definitively prove certain properties of a game, rather than simply showing that they are unlikely.

\footnotetext{
${ }^{1}$ http://decreasoner. sourceforge.net/csr/ecas/
}

\section{The Logical Game Engine}

There some drawbacks to modeling games directly in EC+ASP. In particular, nearly all of the $T$ variables are superfluous because, outside of the event calculus axioms, the logical rules of a game tend to refer only to the current time. Secondly, crafting more complex games directly in the event calculus formalism leads to duplication of common preconditions for an event in each of its initiates/terminates clauses. This duplication creates a maintenance challenge for the game's author, who might want to make simple changes to the conditions for a particular event. Finally, expressing the fact that some set of game events conflict with each other, despite being independently possible, requires mapping a complex idiom (of threading special conditions through most game logic) over each new game design. Mutually conflicting events are a common occurrence even in simple turn-based games, but describing this constraint directly is, in our experience, error-prone. These annoyances are resolved in our engine while retaining the advantages of EC+ASP.

Programming computer games is an immensely difficult task, and only through the continued application of software engineering practices has a sequence of increasingly powerful game engines made possible the rich games we expect today [13]. Game engines attempt to provide standard solutions for game programming problems without prescribing particular rules or setting for a game. These solutions are exposed by a set of APIs that games can be programmed against. Some engines, such as Torque ${ }^{2}$ and Unreal, ${ }^{3}$ go so far as to provide custom programming languages to ease the integration of a game's specification with the engine's services. LUDOCORE is modeled after this richer variant of game engines: it provides not only APIs that encapsulate solutions to difficult logical modeling problems (such as conflicting events) but effectively provides a new language that is tailored to the application of specifying logical game worlds. Games produced with our engine are not only smaller than their equivalent specification using only the event calculus, but also easier to maintain throughout meaningful design changes, and easier for the game's author (or even automated tools) to analyze.

Our game engine is essentially a background theory for logical game descriptions. In the rest of this section we will describe the logical predicates of our game engine and how individual games can leverage them. Figure 2 gives an overview of how our engine builds on the event calculus, and in turn supports modeling games on top of it. The event calculus axioms provide the base semantics for discussing state and events over time. Our engine adds higher-level abstractions for modeling games than the raw event calculus primitives do (discussed below).

To produce a completely specified logical game that admits automatic gameplay trace generation, the author adds a particular game's rules: the specific state and events that make up the game, the consequences of events happening

\footnotetext{
${ }^{2}$ http: / / www. torquepowered. com

${ }^{3}$ http: / / www . unrealtechnology . com
} 


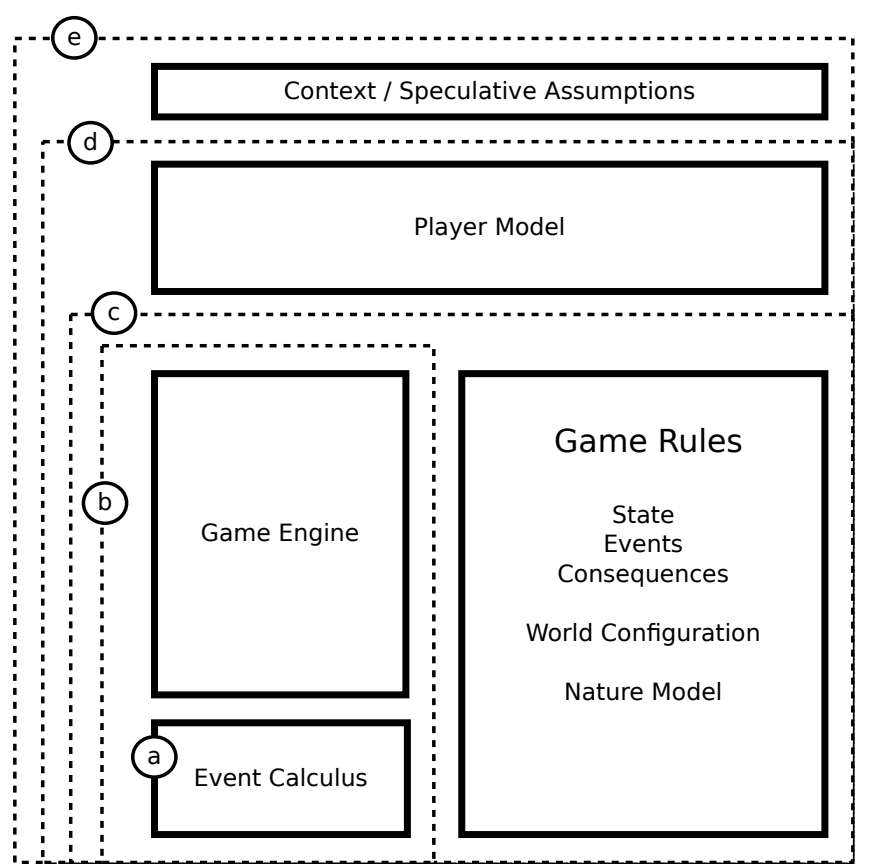

Fig. 2. Block diagram illustrating how the logical theories involved in a complete application of LUDOCORE fit together to form a logic program: (a) provides a temporal logic basis; (b) expands the temporal logic to include videogame-level concepts; (c) is a complete specification of a particular game; (d) provides a model of a certain class of players playing this game; and (e) represents a focused view of particular situations that could arise in this player's play.

in the game world, the configuration of entities within the game world (e.g. level layouts), and a model of when things take place in the game world without the player's intervention (caused by "nature"). While this fully specifies a game world, a player model additionally adds assumptions about what kinds of actions a player can take, in which combinations-either due to actual restrictions (e.g. if the author has in mind an input mapping ${ }^{4}$ that would make certain actions impossible to perform simultaneously), or due to a desire to investigate certain kinds of player behavior. Finally, speculative assumptions can be added that restrict the generated traces to certain kinds of situations that the author wants to investigate.

\section{A. State, events, and consequences}

Since state and events are natural elements of a game's definition, we expose the event calculus's fluents and events in our game engine with the predicates game_state and game_event, respectively. A game state assertion in LUDOCORE looks like this:

$$
\text { game_state }(\operatorname{at}(A, R)) \leftarrow \operatorname{agent}(A), \operatorname{room}(R) .
$$

This assertion reads that at is conceptually a table that records the relation between agents and rooms (answering, “is agent $A$ in room $R$ ?"). Elements of this table stay set

\footnotetext{
${ }^{4}$ We discuss elsewhere [14] why, when modeling games, input mappings make sense to model separately from the mechanics that define a gameworld.
}

until changed otherwise, a property inherited from the event calculus's commonsense law of inertia.

For state that should be updated dynamically as a function of other state, we provide the state_helper mechanism, which provides a computed view on inertial state. State helpers are, semantically, event-calculus fluents with inertia disabled, which cannot be directly initiated or terminated. This stratification into primary and derived fluents that we enforce syntactically is one safe idiom for avoiding the ramification problem in the event calculus [15].

The state_helper assertion below provides a convenient view on the at state for checking when a particular agent is at their starting location (starts_in) without having to name that location. This example also illustrates how rules can be conditioned on the current game state using the holds predicate. This state helper rule implies that home is a dynamic (time-varying) property of the game world:

$$
\begin{aligned}
& \text { state_helper }(\text { home }(A)) \leftarrow \\
& \quad h o l d s(\operatorname{at}(A, R)), \text { starts_in }(A, R) .
\end{aligned}
$$

Game-event declarations are relatively straightforward. The assertion below describes the event of an agent performing a healing action:

$$
\text { game_event }(\text { heals }(A)) \leftarrow \operatorname{agent}(A) .
$$

However, game events can have significantly more structure in LUDOCORE than in the event calculus alone. For example, the conditions under which a game event is possible (e.g., the legality of moves in a board game) are specified using the possible predicate. In this example, agents may only heal in their starting room:

$$
\text { possible }(\text { heals }(A)) \leftarrow \text { home }(A) .
$$

Possibility assertions are used not only for defining the rules of the game, but also to control event selection when generating gameplay traces. Importantly, possibility is deeply intertwined with the event conflict mechanism. An assertion like the one below can keep two otherwise possible events from happening together:

$$
\text { conflicts (heals }(A), \text { moves }(A, R)) \text {. }
$$

By providing possible and pairwise conflicts conditions for game events in a game model, the required eventselection logic can be implemented and debugged once in the game engine, instead of by each game author. By default, actions that are not marked to conflict are safe to co-occur (whereas GDL enforces exactly one player action per turn, which is unsuitable for modeling general videogames).

Game events can be tagged by the game's author with whether they are direct player actions or spontaneous actions that can only be caused by a non-player entity, thought of as "nature" or "the game master". The player_event and nature_event predicates signify this tagging. Although these annotations do not correspond to what we normally think of as game rules, they play an important role in 
scoping the applicability of player-modeling rules, described with the player_asserts and player_forbids predicates (and corresponding nature predicates), discussed later.

Linking game state to game events, the initiates and terminates predicates from the event calculus are exposed to game authors with minimal change. The only modification our engine uses is that these predicates are defined in a timeinvariant fashion, always referring to the current game state. The example below asserts that the moves event causes an update to the at game state, and that the hits event causes the target of the hit to no longer be alive, under certain conditions:

$$
\begin{aligned}
& \text { initiates }(\text { moves }(A, R), \text { at }(A, R)) \text {. } \\
& \text { terminates }\left(\operatorname{hits}\left(A_{1}, A_{2}\right) \text {, alive }\left(A_{2}\right)\right) \leftarrow \\
& \quad \text { holds }\left(\operatorname{armed}\left(A_{1}\right)\right), \text { not holds }\left(\operatorname{armed}\left(A_{2}\right)\right) .
\end{aligned}
$$

Finally, the initial state of the game world is given by, simply enough, the initially predicate, which is often conditioned on world configuration. Even in the examples above, we have suggested the existence of room and starts_in predicates as background knowledge used to control our state-and-event logic. Many predicates of this kind can be thought of as specifying a static world configuration: timeinvariant facts about the game world, such as level geometry, item properties, and tables of weapon effects.

\section{B. Player model interface}

In addition to tagging the subset of game events that are player actions with player_event, we build an additional interface for specifying models for players' behavior.

Simply allowing a certain set of events to be considered by the player, by tagging them as player events, does not immediately yield an expressive modeling interface. The player_asserts and player_forbids predicates can be used to express stronger preferences that an action be taken or should never be taken under any circumstances (forbids beats asserts in our engine). The example below illustrates these assertions in the context of a hypothetical game in which picking up objects is a player action:

$$
\begin{aligned}
& \text { player_asserts }(\text { pickup }(O)) \leftarrow \operatorname{kind}(O, \text { gold }) \text {. } \\
& \text { player_forbids }\left(\text { pickup }\left(O_{1}\right)\right) \leftarrow \\
& \quad \operatorname{kind}\left(O_{1}, K\right), \operatorname{holds}\left(\operatorname{carrying}\left(O_{2}\right)\right), \operatorname{kind}\left(O_{2}, K\right) .
\end{aligned}
$$

This model describes a player who never misses a chance to pick up a gold object, but never picks up duplicates (even of gold objects).

The combination of such assertions allows for the specification of a complex, nondeterministic player action policy that automatically respects the game's mechanics. The default player model is maximally permissive (it reads that the player considers all player events) which allows meaningful play traces to be extracted from games even when no effort has been put into player modeling. Every clause of player_forbids or player_asserts serves to pare down the space of gameplay traces to those that are more reasonable to expect, given the provided knowledge.
Identical in structure to the player modeling interface, we provide nature_asserts and nature_forbids predicates to operate on the events marked with nature_event. Whether the nature model is used to model an opponent, a game master, a collection of non-player characters, or even used at all, is largely a game author's choice. Many of the rules that end up in a nature model could be pushed into the game's core rules, resulting in an equivalent logical model. Leaving certain elements of the game in the realm of the nature model, however, makes it easy to experiment with modification to these policies without modifying the accepted base rules. Having such flexibility is crucial when modeling videogames with a live world, full of enemies, moving platforms, and other active, non-player entities.

\section{Relation to the general event calculus}

In comparison to the general event calculus, we disallow holds and happens from ever being used at the head of a rule. In event-calculus terms, that means we disallow state constraints and triggers.

In general, state constraints are not game mechanics. For example, the state constraint that the hero is never at the bottom of a pit while alive might be true of a bit of design, but it is not itself a game rule. There may be rules that produce this state constraint: for example, saying that the hero dies when he hits the bottom of pits (a terminates clause); and so would a rule that prevented the hero from ever falling off ledges into pits (a possible clause). The result is that every state is either only changed by initiates and terminates, or is known to be completely passive, in the case of state helpers. Disallowing general state constraints ensures that games have well-defined, consistent, procedural meanings (which is required for them to be human-playable).

By disallowing a game's author from directly specifying when an event happens, we can ensure that our engine has complete control over event management, allowing us to implement possible, conflicts, and the player and nature models. The logic required to implement the required semantics of these predicates is highly circular and counterintuitive. Since such a mechanism is required for almost any interesting videogame model, by implementing it once and for all in LUDOCORE, a game author doesn't have to create an ad-hoc reimplementation of similar constructs for each game they model.

While we disable rules with happens at the head, our engine permits a conceptual equivalent to the use of triggers (rules that specify that an event happens whenever a particular combination of state holds) through the nature model. For example, to model a triggered collision event, the nature model could include a line that says nature_asserts the collision event between two objects if their position is identical.

In summary, our engine provides a modified view of the event calculus that is designed to make game-level assertions easy to express while minimizing the possibility of a game author (modeler) accidentally introducing purely logical bugs such as deductive loops and contradictions. This 
lets designers focus on whether their set of game rules is an interesting game, and how it functions, as opposed to spending much time worrying about whether their set of logical assertions specifies a game at all. It should be clear from Figure 2 that a complete, inspectable model of play includes much more than just the event calculus.

\section{LUDOCORE IN ACTION}

Having defined the major ideas in our game engine, we now use it to generate interesting gameplay traces, and to perform incremental rule modification, world configuration changes, and player modeling.

\section{A. Gameplay Trace Inference}

The simplest use of our engine is to simply generate any gameplay trace compatible with a game's definition. One of the difficulties for game designers is understanding the potential consequences of rule interactions. When initially crafting game models, these unconstrained traces provide quick access to possible interactions within the game, quickly revealing undesirable behaviors resulting from game-design bugs. The output of our tools provides not only a $\log$ of game events, but the complete game state at all times, to ease diagnosis.

Figure 3 shows an automatically generated event trace from the LUDOCORE model of a popular online game, Motherload, ${ }^{5}$ in which a mining robot recovers ore and treasures from a network of caverns without running out of fuel (our playable model of the game is illustrated in Figure 1). In this case, we show both player-action events, such as mine(al) (the player's robot mined the rock named $a 1$, which removed it from the world and added it to the robot's inventory), and natural events, such as drain (the robot's energy drains by one unit). The player here mines a single rock reachable from the starting location, and trades it in and refuels. Then, he embarks on a longer mining journey, mining a few rocks and moving downwards into the ground, before finishing with some fairly aimless wandering up and down. The values of each piece of game state at each time step can also be viewed, but we focus here on just the event trace.

While Figure 3 shows a random trace with no constraints, the most common use of LUDOCORE is combining a game with a set of hypothetical or speculative assumptions (SAs). SAs are commonly used to assert that certain events do happen or never happen, or that a certain condition is met by a certain timepoint (e.g., victory within 20 turns). This helps explore the space in a much more focused way than simply looking at random gameplay traces; for example, while the random wandering up and down in Figure 3 is a common feature of random traces, it doesn't illustrate anything particularly interesting about the gameplay possibilities.

The mechanism of specifying speculative assumptions (based on integrity constraints) provides a simple, modular querying interface for narrowing down to more interesting

\footnotetext{
$5_{\text {http: //www.xgenstudios.com/play/motherload }}$
}

\begin{tabular}{|c|c|}
\hline happens ( & mine (a1), \\
\hline happens ( & drain, \\
\hline happens ( & drain, \\
\hline happens ( & trade, \\
\hline happens ( & refuel, \\
\hline happens ( & mine (a2), \\
\hline happens ( & mine $(\mathrm{a} 0)$ \\
\hline happens ( & down_to (a), \\
\hline happens ( & mine (space_canary_corpse), \\
\hline happens ( & $\operatorname{mine}(\mathrm{c} 0)$ \\
\hline happens ( & down_to (c), \\
\hline happens ( & down_to $(f)$, \\
\hline happens ( & up_to (c), \\
\hline happens ( & up_to (a), \\
\hline happens ( & down_to (c), \\
\hline happens ( & down_to (f), \\
\hline
\end{tabular}

Fig. 3. Example gameplay trace for the game shown in Figure 1, generated by asking our analysis engine for a random 15-time-step trace with no constraints.

traces, and asking questions about gameplay possibilities. "Is my game winnable?" requires only one SA to specify (asserting that victory happens at some timepoint). To scope the question further, asking "is my game winnable with avatar health never dropping below 5?", requires only one more SA. In our model of Motherload, we used SAs to look at extreme kinds of gameplay possibilities: speed runs by players beating the game in as short a time as possible, or what kinds of gameplay would result if a player never refuelled, or refuelled cautiously. We compared these traces to traces from human playtesters, who gave us an idea of how beginners would play the game before being familiar with it, which showed us, for example, that our players were much more cautious on refuelling than necessary. ${ }^{6}$

In generating these kinds of constrained traces, the logical inference approach taken in EC+ASP shines. Had we used randomized forward search in the game's state space, constraints on happenings of the final timepoint would be difficult to encode and inefficient to compute, requiring exhaustive search to prove nonexistence. ASP lets us "run the game backwards" or even sideways ${ }^{7}$ as needed to satisfy trace constraints or quickly prove them impossible.

While gameplay traces can be highly informative (because each includes a complete narrative of events), even the absence of traces can be informative. If adding a particular $\mathrm{SA}$ yields no traces for a game that previously admitted many, then it has been proven (by contradiction) that the SA is incompatible with the game's rules, i.e. that the assumption is false in the game's abstract world. ${ }^{8}$

Extraction of gameplay traces is the primary function of the LUDOCORE engine. The affordances we describe below

\footnotetext{
${ }^{6}$ We discuss this complementary use of human and machine playtesting in more detail elsewhere [1].

${ }^{7}$ Inferring which state is compatible with a particular narrative of events

${ }^{8}$ Thielscher [16] also uses termination without models of an ASP solver to prove properties of a game via contradiction.
} 
for modeling and modifying games all serve to give the game's author the ability to sculpt the space of traces that our system will generate.

\section{B. Modifying rules and configuration}

Recall that game design is often an iterative process, adding, removing, and tweaking rules as a design progresses. Rules, in LUDOCORE, are represented by the game_event, game_state, possible, conflicts, and initiates/terminates predicates. A game's author might make changes to these rules (and examine the traces for the new game) as part of a major intentional design change, or simply as a way of quickly testing the implications of alternative formulations of a particular mechanic in the game. Often it is useful to disable (by simply commenting-out) several rules to focus on sub-parts of a game in isolation without distracting interactions, e.g. disabling ammunition consumption on weapons when examining health-point management. In a language without elaboration tolerance, this would require more complex editing than simply commenting out a rule. Creating a variant of chess without castling in GDL, for example, cannot be done simply by removing the castling rule, but requires other edits as well.

To illustrate rule modifications, we draw on two examples from our LUDOCORE model of Motherload (discussed earlier, and shown in Figure 1).

If we wanted to add a fixed inventory capacity to our mining robot (constraining an existing event on the basis of existing game state), we would modify the possible conditions for our game's mine event to depend on a count of the number of items for which the bagged state for rocks held. This single change would not only stop traces that violate the new rule from being generated but reject traces when even an externally assumed narrative included too many mining events:

$$
\begin{aligned}
& \text { state_helper }(\text { have_space }) \leftarrow \\
& \quad \text { count }(R, \text { holds }(\text { bagged }(R)), N), N<10 . \\
& \text { possible }(\text { mine }(R)) \leftarrow \\
& \quad \text { holds }(\text { present }(R)), \text { touching }(R), \text { fueled, have_space } .
\end{aligned}
$$

To modify the game to charge players energy points for mining instead of moving, we would drop the initiates and terminates rules linking the moving event to the energy state and create two new ones linking mining to moving (one to terminate the previous energy value and another to initiate the diminished value). This re-wiring task incurs only a fourline change to the game source when using our engine, due to the conciseness of expressions enabled by our game engine. With access to gameplay trace inference, we can quickly verify the effectiveness of the rule modification without the need for human play testers.

This ease of rule modifications directly derives from the elaboration tolerance afforded by the underlying EC formalism. McCarthy [10] describes elaboration tolerance as the ability (of a knowledge representation) to accept changes to known facts without having to "start over", which is exactly what we realize for the space of games. Further, in other research applying EC to games, we have shown how entire modules (corresponding to game mechanics and vocabularies) can be swapped in and out without trouble [5].

In addition to swapping rules, it is quite easy to pair a fixed game with different sets of configuration such as map layout, static item properties, and tweakable constants. For example, a simple map can be used for early testing, and a more complex one used later for detailed player modeling. In addition to manually specified game configurations, it is possible to let the ASP solver reason across possible configurations for the game. In our model of the game Minesweeper, we are able to reason over possible placements of mines that are consistent with player observations. In a simple dungeon crawl game, we allowed the solver to manipulate the presence and connectivity of rooms on a map. The ability to make structural queries of this sort is a novel feature for game engines.

\section{Using player and nature models}

While we think of speculative assumptions as convenient but throwaway constraints, specifying complex assumptions can be tedious. The player modeling interface provides a more straightforward way of building larger player models that will be retained and modified, rather than used in only a few queries. Sets of player_asserts and player_forbids clauses are collected to carve out the space of behavior that can be reasonably expected of the kinds of player being investigated.

A custom nature model can similarly be used to carve the behavior of other in-game entities. In the dungeon crawl game, we used the nature model to control monster wandering behavior. We had tagged the monster movement event as a nature event, implying that it should always be considered in trace finding when using the default nature model. To effect patrolling behavior, we used the following assertion, which yields monsters who wander only within their own marked areas:

$$
\begin{gathered}
\text { nature_forbids }\left(\text { move_to }_{-}(M, R)\right) \leftarrow \\
\text { not patrolled_by }(M, R) .
\end{gathered}
$$

\section{Applications EnABLED}

LUDOCORE enables a number of broader applications, some of which have already been investigated in other forms.

\section{A. Game design}

Designing games in a logical game engine, or at least using one to prototype game ideas, can provide designers with insight about design pros and cons, and suggest possible improvements. Many logical formalisms can allow verification that desired properties hold, and undesired properties don't. However, designers often work in an iterative, exploratory manner, and find exact yes-or-no queries somewhat difficult to formulate [17]. The experimentation with gameplay traces that our engine supports can help designers understand the possibilities of their design by iteratively "zooming in" on 
specific kinds of traces using SAs, and observing how different player models shape emergent properties of the game. We have already begun pursuing this application, creating a game prototyping system [1] in which human-playable, board-game-like prototypes can be built, using the logical representations described in this paper. Figure 1 shows an example designed as if to be an early prototype version of Motherload (discussed earlier). This prototype focuses on the core mechanics: moving around underground, mining, and refueling (whereas Motherload also includes shopping for upgrades and story elements). These prototypes support two kinds of playtesting: traditional observations of human players, and a new mode of machine playtesting, in which observation of machine-generated gameplay traces is the focus.

Logical game engines may also form a core component of emerging research in automated videogame design. In a nonlogical approach, Togelius and Schmidhuber [18] generate Pac-Man variants from a parameterized space and use reinforcement learning to demonstrate gameplay in the resulting games. Games implemented in LUDOCORE can be varied in a much more open-ended and incremental manner than with a parameterized space of variation, and its elaboration tolerance addresses the problem of brittleness of symbolic representations, which was in part responsible for the move towards parameterized numerical representations in contentgeneration research.

Furthermore, given sets of traces, inductive logic programming (ILP) can be used to induce models for the style of gameplay exhibited in those traces. The player modeling interface in LUDOCORE makes this more feasible by having player models built from only two key predicates. Many popular ILP systems, such as Progol [19] can only learn single predicates at a time. In our player modeling interface, single-predicate ILP systems can learn the player_asserts predicate from example gameplay traces. Some research has even been done on learning EC rules themselves [20], which, for games, would open up the possibility of inducing new game rules from a collection of desired gameplay traces.

Procedural level design can be done by specifying and solving constraints for what constitutes a good level, sometimes as part of a larger search process [e.g. 21]. Particularly relevant here, a community-driven project recently added procedurally generated maps and base layouts to the realtime strategy game Warzone 2100, using ASP to specify and solve constraints on the maps' layouts. ${ }^{9}$ Designing a good level, however, often means designing a level that supports good gameplay, and these sorts of static constraints only indirectly speak to gameplay. Other approaches, such as that of Togelius et al. [22], generate levels and score them with a fitness function that predicts whether they support interesting gameplay. For games written using our logical game engine, a constraint-based approach can easily include these kinds of constraints on gameplay in addition to constraints on static level properties, because the level-generation search and

\footnotetext{
${ }^{9}$ http://warzone2100.org.uk/manual-diorama
}

game-tree search are unified into the same query mechanism.

\section{B. Crafting game playing agents}

Despite our motivating focus on analyzing game designs, our logical game engine has applications to playing games as well. Because gameplay trace inference in our engine corresponds exactly to what is commonly known as EC planning [23], it is possible to use our engine directly in a general game player. Thielscher [16] already argued for the applicability of ASP with temporal-logic models for the contemplation phase of GGP competitions.

The player modeling interface we provide is designed to accept incremental additions of knowledge about how a player (or their opponents) make choices. Though this interface cannot express a minimax-like policy (that includes quantification over models at every timepoint), it does coalesce facts into a unified move-set selection policy which would allow minimax to operate in the more restricted space in which the modeled opponent is really playing.

The ease of adding and removing rules in our games has another benefit for those wishing to craft general game players. The elaboration tolerance of the representation makes syntactic construction of novel games much easier. By building all combinations of a fixed set of add-on mechanics, a generative space of games can be realized, providing a much wider selections of games for agents to be tested on.

\section{CONCLUSION}

In this paper, we introduce a new concept: the logical game engine. Our logical game engine, LUDOCORE, much like a traditional game engine, both provides a higher-level language to describe games, and centralized solutions to tedious or error-prone programming tasks. By virtue of using logic, we gain not only a concise representation of a game's mechanics, but also the ability to automatically generate interesting gameplay traces that meet meaningful constraints.

While clearly LUDOCORE serves as a bridge from the concerns of game design to logic-based AI tools, it has also served as the basis for implementing interactive prototypes. In BIPED [1], human-playtestable prototypes with real-time interaction and graphics are written with the LUDOCORE engine, enabling the same game specifications to both be used with logical tools (objective, machine playtesting) and as gameplay demos (subjective, human playtesting).

Apart from demonstrating its utility by building BIPED on it, the question of how to evaluate LUDOCORE itself is more subtle. We are not immediately interested in metrics such as benchmarks. Rather, the primary driver of our research is expressiveness, the ability for human or automated game designers to succinctly specify and informatively query models of gameplay. We want to answer questions such as: How easy is it to specify different kinds of games? Is making common kinds of modifications to a game easy? Are there queries we can't answer from this kind of model? We've designed with these questions in mind, but a satisfying answer to these questions must come from the kind of extensive case studies 
that look at the evolution of a multiple game designs through many stages of modification.

Future work on LUDOCORE will aim to further improve the process of specifying, working with, and reusing formal game models. Although the event calculus' elaboration tolerance gives specifications some built-in modularity, a higherlevel module system would make it easier to quickly swap in and out larger subsystems, such as pre-specified inventory systems or economy models. Language improvements can also simplify the specification of many common kinds of game mechanics, for example by adding inheritance between game objects, or higher-level handling of numerical operations. Finally, our goal in building LUDOCORE is to enable two main applications: an automated game design system that can understand and manipulate game rules [24], and a game-design prototyping system that provide designers quick feedback and deeper insight into early-stage designs [17].

\section{REFERENCES}

[1] A. M. Smith, M. J. Nelson, and M. Mateas, "Computational support for play testing game sketches," in Proceedings of the 5th Artificial Intelligence and Interactive Digital Entertainment Conference (AIIDE), 2009.

[2] N. Love, T. Hinrichs, D. Haley, E. Schkufza, and M. Genesereth, "General game playing: Game description language specification," Stanford Logic Group, Tech. Rep. LG-2006-01, 2006.

[3] M. Genesereth, "General game playing: Overview of the AAAI competition," AI Magazine, vol. 26, no. 2, pp. 62-72, 2005.

[4] T. Fullerton, Game Design Workshop. Morgan Kaufmann, 2008.

[5] M. J. Nelson and M. Mateas, "Recombinable game mechanics for automated design support," in Proceedings of the 4th Artificial Intelligence and Interactive Digital Entertainment Conference (AIIDE), 2008, pp. 84-89.

[6] E. Adams, Fundamentals of Game Design. New Riders, 2009.

[7] M. Shanahan, Solving the Frame Problem: A Mathematical Investigation of the Common Sense Law of Inertia. MIT Press, 1997.

[8] E. T. Mueller, Commonsense Reasoning. Morgan Kaufmann, 2006.

[9] E. T. Mueller, "Event calculus reasoning through satisfiability," Journal of Logic and Computation, vol. 14, no. 5, pp. 703-730, 2004.

[10] J. McCarthy, "Elaboration tolerance," in Proceedings of the 4th Symposium on Logical Formalizations of Commonsense Reasoning, 1998.

[11] T.-W. Kim, J. Lee, and R. Palla, "Circumscriptive event calculus as answer set programming," in Proceedings of the 21st International Joint Conference on Artificial Intelligence (IJCAI), 2009, pp. 823-829.
[12] V. Lifschitz, "What is answer set programming?" in Proceedings of the 23rd National Conference on Artificial Intelligence (AAAI), 2008, pp. 1594-1597.

[13] J. Blow, "Game development: Harder than you think," ACM Queue, vol. 1, no. 10, pp. 28-37, 2004.

[14] M. J. Nelson and M. Mateas, "Towards automated game design," in AI*IA 2007: Artificial Intelligence and Human-Oriented Computing, 2007, pp. 626-637.

[15] M. Shanahan, "The ramification problem in the event calculus," in Proceedings of the 16th International Joint Conference on Artificial Intelligence (IJCAI), 1999, pp. 140-146.

[16] M. Thielscher, "Answer set programming for singleplayer games in general game playing," in Proceedings of the 25th International Conference on Logic Programming (ICLP), 2009, pp. 327-341.

[17] M. J. Nelson and M. Mateas, "A requirements analysis for videogame design support tools," in Proceedings of the 4th International Conference on the Foundations of Digital Games (FDG), 2009, pp. 137-144.

[18] J. Togelius and J. Schmidhuber, "An experiment in automatic game design," in Proceedings of the 2008 IEEE Symposium on Computational Intelligence and Games (CIG), 2008, pp. 111-118.

[19] S. Muggleton, "Inverse entailment and Progol," New Generation Computing Journal, vol. 13, pp. 245-286, 1995.

[20] S. Moyle and S. Muggleton, "Learning programs in the event calculus," in Proceedings of the 7th International Workshop on Inductive Logic Programming, 1997, pp. 205-212.

[21] G. Smith, M. Treanor, J. Whitehead, and M. Mateas, "Rhythm-based level generation for 2D platformers," in Proceedings of the 4th International Conference on the Foundations of Digital Games (FDG), 2009, pp. 175-182.

[22] J. Togelius, R. De Nardi, and S. M. Lucas, "Towards automatic personalised content creation for racing games," in Proceedings of the 2007 IEEE Symposium on Computational Intelligence and Games (CIG), 2007, pp. 252-259.

[23] M. Shanahan, "Event calculus planning revisited," in Proceedings of the 4th European Conference on Planning, 1997, pp. 390-402.

[24] A. M. Smith and M. Mateas, "Variations Forever: Flexibly generating rulesets from a sculptable design space of mini-games," in Proceedings of the 2010 IEEE Conference on Computational Intelligence and Games (CIG), 2010. 